\title{
High-Speed Electronics for Short-Link Communication
}

J. Bauwelinck ${ }^{(1)}$, R. Vaernewyck ${ }^{(1)}$, J. Verbrugghe $^{(1)}$, W. Soenen ${ }^{(1)}$, B. Moeneclaey ${ }^{(1)}$, C. Van Praet ${ }^{(1)}$, A. Vyncke ${ }^{(1)}$, G. Torfs ${ }^{(1)}$, X. Yin ${ }^{(1)}$, X.Z. Qiu ${ }^{(1)}, J$. Vandewege ${ }^{(1)}$, N. Sotiropoulos ${ }^{(2)}$, H. de Waardt ${ }^{(2)}$, R. Cronin $^{(3)}$, G. Maxwell ${ }^{(3)}$, T. Tekin $^{(4)}$, P. Bakopoulos ${ }^{(5)}$, C.P. Lai( ${ }^{(6)}$, P.D. Townsend ${ }^{(6)}$

(1) INTEC/IMEC, Ghent University, Sint-Pietersnieuwstraat 41, 9000 Gent, Belgium, johan.bauwelinck@intec.UGent.be

(2) COBRA Institute, Eindhoven University of Technology, NL-5600MB Eindhoven, The Netherlands

(3) CIP Technologies, Adastral Park, Marlesham Heath, Ipswich, IP5 3 RE, UK

(4) Fraunhofer IZM, D-13355 Berlin, Germany

(5) School of Electrical \& Computer Engineering, National Technical University of Athens, Greece

(6) Photonic Systems Group, Tyndall National Institute, University College Cork, Ireland

Abstract High-speed electronic integrated circuits are essential to the development of new fiber-optic communication systems. Close integration and co-design of photonic and electronic devices are becoming more and more a necessity to realize the best performance trade-offs. This paper presents our most recent results and a brief introduction to our research in recently started EU projects.

\section{Introduction}

Optical data transmission is the key to highbandwidth energy-efficient communication to satisfy the ever increasing capacity demands of today's ICT infrastructure. Commercial fiberoptic solutions are already well established in long-reach core, metro and access networks, while research in these fields continues to make impressive progress. Nowadays, fiber-optic technology is also entering shorter-reach applications supported by intensive research programs on e.g. datacenter and highperformance computing systems such as optical backplanes and active optical cables.

Thanks to the miniaturization of photonic technologies and advances in packaging, lowpower consumption and very high bandwidth components are becoming available which will gradually replace purely electrical links as their practical limits are reached. Nevertheless, highspeed electronic circuits remain crucial to the success of optical interconnections, not only to generate, process and store huge amounts of data, but also to interface between two very different worlds: the purely digital CMOS and the rather analog opto-electronic ( $\mathrm{E} / \mathrm{O}$ and $\mathrm{O} / \mathrm{E}$ ) devices, e.g. lasers/modulators and their respective drivers as well as photodiodes and receivers. These high-speed opto-electronic devices are the main topic of this paper.

\section{Co-design of electronics and photonics}

Dedicated analog or mixed-signal circuits are needed to obtain the best cost-performancepower trade-off, taking into account the system requirements, modulation scheme and photonic components used. The most critical point is the interconnection between driver and modulator/laser and between photodiode and transimpedance amplifier (TIA). First of all, bringing electronics and photonics close together is important to minimize electrical parasitics (e.g. inductance and capacitance) that would otherwise reduce the bandwidth or require transmission line interconnects (with considerable power dissipation in their termination resistors). Excessive parasitics can also strongly impact the stability and, consequently, the sensitivity of the TIA. Due to the dense integration, it is often impossible to integrate a bias tee in between the electronic

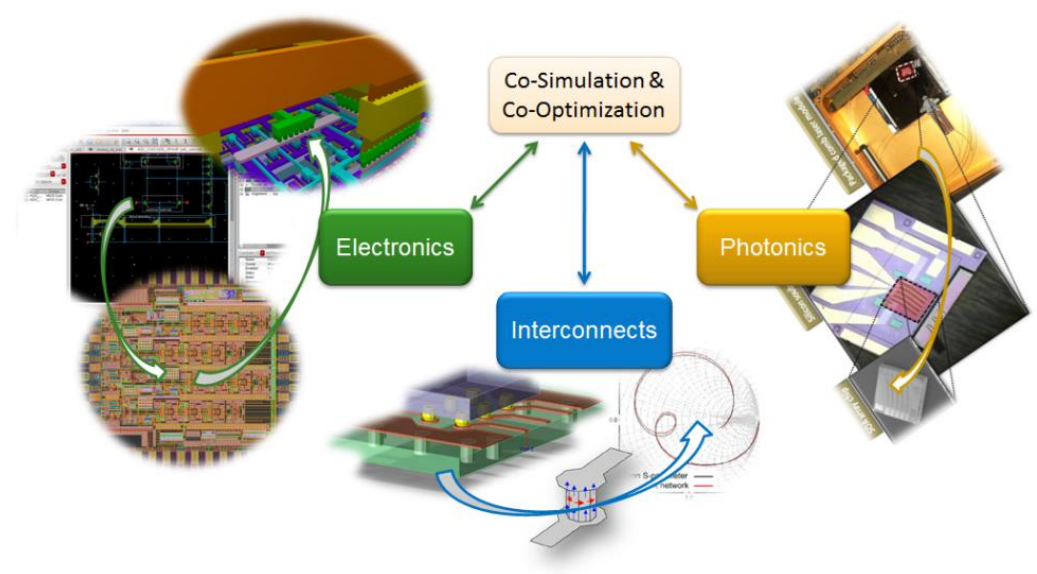

Fig. 1: Co-simulation and co-optimization of electronics, photonics, and interconnects 
and photonic components, requiring a DCcoupled design. Additionally, compact, lowpower and low-cost multi-channel transmitter and receiver modules are an absolute necessity for high-speed short-links. The close integration of several driver and TIA circuits on a single chip poses several design challenges e.g. low power consumption to alleviate thermal issues, small footprint and low RF crosstalk.

Fig. 1 schematically represents a co-simulation and co-optimization process of electronics, photonics and interconnects. Typically, each aspect requires a dedicated simulation tool. The resulting equivalent models, however, can be imported in a professional integrated circuit (IC) design environment such as Cadence Virtuoso, Mentor IC station or Agilent ADS to run cosimulations. In this way, there is no need for a pre-defined impedance level (typ. 50Ohm) for the RF interfaces because high-speed electronics and photonics can be co-optimized with interconnect parasitics.

28Gb/s low-power EAM driver arrays for inter-datacenter point-to-point links

Within FP7 ICT project C3PO ${ }^{1}$, we developed a $10 \times 11 \mathrm{~Gb} / \mathrm{s}$ electroabsorption modulator (EAM) driver array, a $2 \times 28 \mathrm{~Gb} / \mathrm{s}$ duobinary EAM driver array and a $4 \times 28 \mathrm{~Gb} / \mathrm{s}$ TIA array in a $130 \mathrm{~nm}$ SiGe BiCMOS technology. It was shown that the concurrent design improves optoelectronic performance by co-optimising the key parameters of these $E / O$ and $O / E$ devices. In particular, the co-design of the driver arrays, the interconnection to the EAM and the high (250Ohm) drive impedance allows for a drastic reduction of the $10 \times 11 \mathrm{~Gb} / \mathrm{s}$ driver array's power consumption (merely $2.2 \mathrm{~W}$ in total or $220 \mathrm{~mW}$ per $11 \mathrm{~Gb} / \mathrm{s}$ channel at $2.5 \mathrm{Vpp}$ single-ended drive) $)^{2}$.

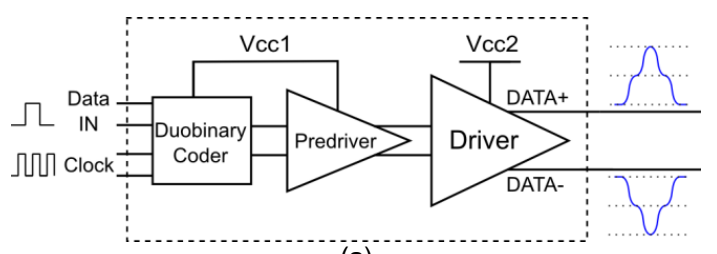

(a)

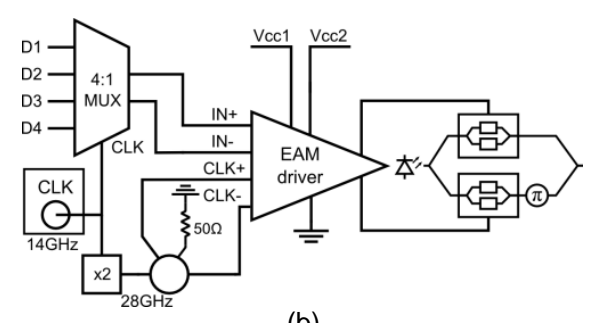

(b)

Fig. 2: (a) Simplified duobinary driver block diagram (b) Test setup with nested Mach-Zehnder modulators

For the 28Gb/s duobinary driver, shown in Fig. 2 (a), the focus was not only on power consumption reduction, but also on the efficient and reliable on-chip conversion of NRZ data to duobinary signals. The selected technique is robust against temperature, process and supply variations and allows operation over a range of data rates, which is not possible with conventional approaches based on low-pass filters $^{3}$. The electrical test board, without optical modulators, is shown in Fig. 3. Clean eye diagrams were obtained for differential drive voltages from $3 \mathrm{Vpp}$ up to 6Vpp, as shown in Fig. 4. Though the duobinary encoder and precoder contribute $120 \mathrm{~mW}$ to the power consumption, the IC power-per-bit performance remains well below the state-of-the-art (i.e. $650 \mathrm{~mW} /$ channel at $6 \mathrm{Vpp}, 330 \mathrm{~mW}$ at $3 \mathrm{Vpp})$.

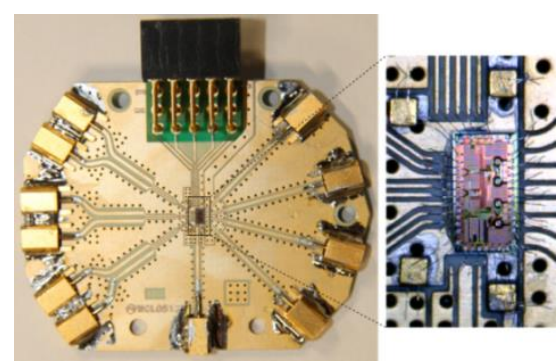

Fig. 3: Two channel $28 \mathrm{~Gb} / \mathrm{s}$ duobinary driver electrical test board and die micrograph

To generate an optical duobinary signal, a Fujitsu nested Mach Zehnder modulator was used as shown in Fig. 2(b). The optical duobinary signal was decoded by a $20 \mathrm{GHz}$ $83440 \mathrm{C}$ photodiode, restoring the original $28 \mathrm{~Gb} / \mathrm{s} \mathrm{NRZ}$ eye diagram, shown in Fig. 5. From this experiment, it is expected that the ongoing integration with a more efficient EAM-based duobinary transmitter will give excellent results as well ${ }^{4}$.

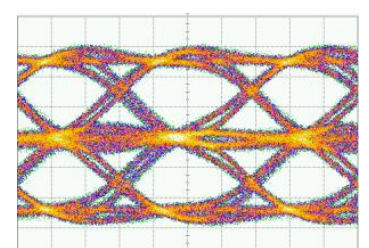

(a) $3 \mathrm{Vpp}, 300 \mathrm{mV} / \mathrm{div}$

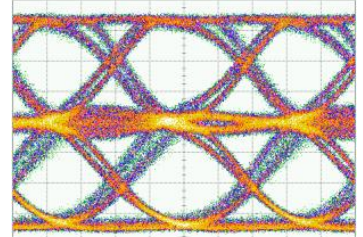

(c) $5 \mathrm{Vpp}, 632 \mathrm{mV} / \mathrm{div}$

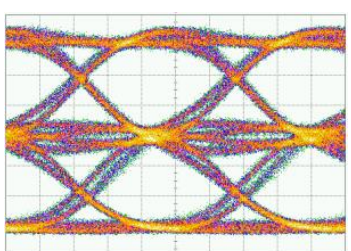

(b) $4 \mathrm{Vpp}, 632 \mathrm{mV} / \mathrm{div}$

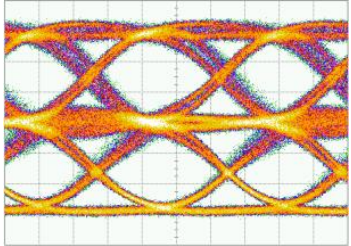

(d) $6 \mathrm{Vpp}, 1 \mathrm{~V} / \mathrm{div}$
Fig. 4: Duobinary 28Gb/s electrical differential drive signals for different outputs (time scale 10ps/div)

VCSEL driver and TIA arrays

VCSEL technology has been the main 
workhorse in optical interconnects due to the inherent low cost, high yield, low power consumption and reliability. Multi-mode VCSELs emitting at $850 \mathrm{~nm}$, as the most mature, have already found their way to commercial applications whereas single-mode longwavelength VCSELs have been identified as a migration step, promising higher modulation speeds and longer reach, essential for future warehouse-scale mega-datacenters and campus networks ${ }^{5}$.

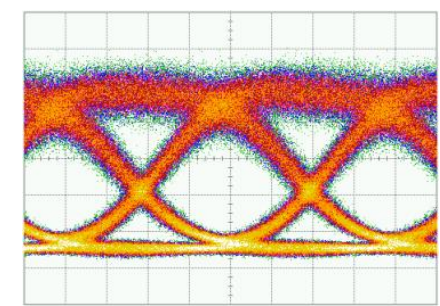

Fig. 5: Back-to-back 28Gb/s eye after O/E conversion (10ps/div, 50mV/div)

The fastest NRZ directly modulated 850nm VCSEL driver reported so far operates at $56.1 \mathrm{~Gb} / \mathrm{s}$ and consumes $682 \mathrm{~mW}^{6}$. Lower power consumption is needed to integrate a considerable number of drivers in an array to cost-effectively scale up the total transmission capacity. Figure 6 shows a simulation of a lowpower driver under development with integrated equalizer. In this simulation, the VCSEL driver circuit was driving a bandwidth-limited VCSEL $(\sim 17 \mathrm{GHz})$ with a $40 \mathrm{~Gb} / \mathrm{s} 2^{7}-1$ PRBS NRZ signal. Fig. 6(a) and 6(b) show the simulated drive current and VCSEL optical output without any equalization. The VCSEL bandwidth limitation results in inter-symbol interference (ISI) and degrades the output signal quality, as shown in Fig. 6(b). By applying a one-tap feedforward equalizer filter, the driver current waveform was pre-distorted (Fig. 6(c)), to improve the highspeed signal quality of the VCSEL output. In Fig. $6(d)$, the output peak-to-peak jitter has been reduced considerably while the vertical eye height improvement is more than $3 \mathrm{~dB}$.

Besides NRZ, PAM-4 modulation is receiving a lot of interests for VCSEL links. For example, recent experiments using long-wavelength VCSELs have demonstrated transmission rates up to $100 \mathrm{~Gb} / \mathrm{s}^{7}$, based on PAM-4 modulation generated with a power combiner, linear amplifiers, a bias tee, FEC and offline equalization. Power-efficient generation of PAM4 on a driver chip with multiple channels requires a different approach, which is also one of our ongoing research activities.

Our VCSEL driver array and associated TIA array designs are being developed within the recently started FP7 projects Phoxtrot $^{8}$ and
Mirage $^{9}$, focusing on cost- and energy-efficient $\mathrm{Tb} / \mathrm{s}$ scale photonic interconnects for data centers and high-performance computing systems.

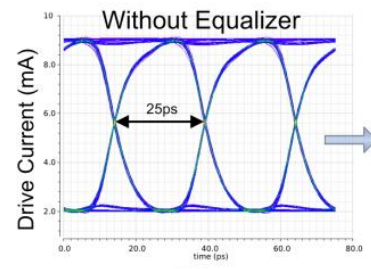

(a)

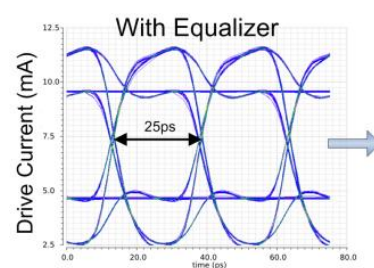

(c)

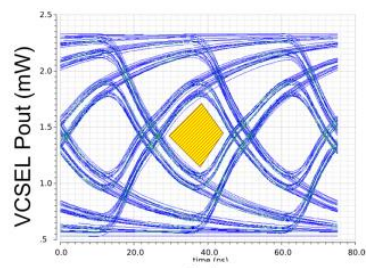

(b)

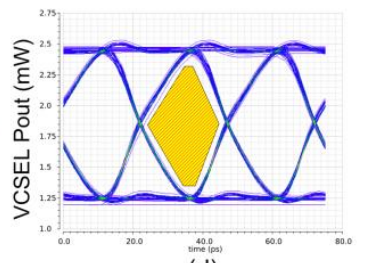

(d)
Fig. 6: Transistor-level simulation of a VCSEL driver with 1-tap FFE equalization: drive current and VCSEL optical output, (a) (b) without FFE (c) (d) with FFE

\section{Conclusions}

This paper discussed various aspects of the design of high-speed electronics for short optical links. Our most recent results of a $2 \times 28 \mathrm{~Gb} / \mathrm{s}$ duobinary driver, with record low power consumption, are presented and some recently started activities are introduced.

\section{Acknowledgements}

This work was supported by the EU-funded FP7 ICT projects C3PO, Mirage and Phoxtrot, the Special Research Fund of Ghent University and the Hercules project VeRONICa for the chip fabrication of the $2 \times 28 \mathrm{~Gb} / \mathrm{s}$ duobinary driver. We also wish to thank Ludo Viaene and Danny Frederickx of IMEC for the chip on board wire bonding and all collaborators not included in the author list.

\section{References}

[1] P. D. Townsend et al., Proc. ECOC'11, Tu.5.LeSaleve (2011).

[2] R. Vaernewyck et al., Proc. ECOC'12, Mo.2.B.2 (2012).

[3] S.K. Kim et al., J. Lightwave Technol., vol. 19, no. 4, pp.465-470 (2001) .

[4] C. P. Lai et al, Proc. ECOC'12, We.1.E.4 (2012)

[5] E. Kapon and A. Sirbu, Nature Photonics, vol. 3, no. 1, pp. 27-29 (2009).

[6] D. M. Kuchta et al., Proc. OFC'13, OW1B.5. (2013).

[7] R. Rodes et al., Proc. OFC'12, PDP5D.10 (2012).

[8] http://www.phoxtrot.eu/

[9] http://www.ict-mirage.eu/ 